\title{
Almost Congruence Extension Property for subgroups of free groups
}

\author{
Lev Glebsky; Nevarez Nieto Saul
}

\begin{abstract}
Let $H$ be a subgroup of $F$ and $\langle\langle H\rangle\rangle_{F}$ denote the normal closure of $H$ in $F$. We say that $H$ has Almost Congruence Extension Property (ACEP) in $F$ if there is a finite set of nontrivial elements $\digamma \subset H$ such that for any normal subgroup $N$ of $H$ one has $H \cap\langle\langle N\rangle\rangle_{F}=N$ whenever $N \cap \digamma=\emptyset$. In this paper we provide a sufficient condition for a subgroup of a free group not possess ACEP. We also show that any finitely generated subgroup of a free group satisfies some generalization of ACEP 1
\end{abstract}

\section{Introduction}

Let $X$ be a group. We use the following standard notations: $Y<X$ for "Y is a subgroup of $\mathrm{X}$ ", $Y \triangleleft X$ for " $Y$ is a normal subgroup of $X$ ", $\langle Y\rangle$ for "the subgroup generated by $Y,\langle\langle Y\rangle\rangle_{X}$ for "the normal closure of $Y$ in $X "$ ". (In the last two cases $Y \subseteq X$.)

Definition 1. Let $F$ be a group. A subgroup $H$ of $F$ has Congruence Extension Property (CEP) if, for any normal subgroup $N$ of $H$, one has $H \cap\langle\langle N\rangle\rangle_{F}=N$.

The CEP is also known by different names. Ol'shanski [2] calls it property $F(n)$, B. H. Newmann [3] names subgroups with the CEP as E-subgroups. Stailings [4] calls them normal convex subgroups. Finally, Osin [5] introduces the self-explaining name CEP. It is worth mentioning that the term CEP was used before for subalgebras and subsemigroups. The natural question is: when does a subgroup $H$ of a group $F$ possess CEP? The question seems difficult, even when $H$ is a finitely generated subgroup of a free group $F$. Particularly, its algorithmic decidability is, as far as we know, an open question. An obvious example of a subgroup with the CEP is a free factor of $F$, and a nontrivial example is given in [6]. Osin [5] introduced the following definition.

Definition 2. Let $F$ be a group. A subgroup $H$ of $F$ has almost $C E P(A C E P)$ if there is a finite set of nontrivial elements $\digamma \subset H$ such that for any $N \triangleleft H$ one has $H \cap\langle\langle N\rangle\rangle_{F}=N$ whenever $N \cap \digamma=\emptyset$.

Henceforth we will use 1 to denote the identity element of the group. An equivalent definition of ACEP is:

Definition 3. A subgroup $H$ of a group $F$ has $A C E P$ if there is a finite set of nontrivial elements $\digamma \subset H$ such that any epimorphism $\theta$ from $H$ to any group $G$ can be extended to an epimorphism $\theta^{*}: F \longrightarrow G^{*}>G$, whenever $\theta(\alpha) \neq 1, \forall \alpha \in \digamma$.

ACEP is a natural and interesting property; moreover, it is easier to find some criteria for determining when a subgroup has ACEP. Our starting point is the following sufficient condition given by D. Osin in [5].

\footnotetext{
${ }^{1}$ Part of this work was done at the Erwin Schrödinger Institute in Vienna, January-March 2016, during the Measured Group Theory program and was partially supported by the European Research Council (ERC) grant no. 259527 of G. Arzhantseva. Part of this work was done at the Nizhny Nivgorod University and supported by the RSF (Russia) grant 14-41-00044. The authors are grateful to the referee for useful suggestion and bringing [1] to our attention.
} 
Definition 4. Let $H$ be a subgroup of a group $F, H^{a}=\left\{a^{-1} h a \mid h \in H\right\}$ with $a \in F$. We say that $H$ is a malnormal subgroup of $F$ if $\forall a \in F \backslash H$

$$
H \cap H^{a}=\{1\} .
$$

We say that $H$ is cyclonormal if $\forall a \in F \backslash H$,

$$
H \cap H^{a}
$$

is a cyclic subgroup.

Theorem 1 (See [5]). Any finitely generated malnormal subgroup of a free group possesses ACEP.

The theorem is Corollary 1.5 of [5] and it is, indeed, a corollary of the main result of [5]. The purpose of our paper is twofold:

- We provide a sufficient condition for a subgroup of a free group not possess ACEP. This condition and the theorem above allow us to "almost decide" when a finitely generated subgroup (f.g.s.) of a free group has ACEP. Still, we are not able to provide an algorithm to decide when an f.g.s. of a free group has ACEP.

- We give further proof of Theorem 1, different from the one provided by [5]. Our proof uses the small cancellation theory and also leads to a more general result, Theorem 3 . Theorem 3 is not a direct consequence of the results of [5] but may be considered as a particular case of Theorem 7.15 in [1]. Our proof gives explicit estimates of some parameters, see Subsection 4.3. It is not clear how to deduce these estimates from (proof of) Theorem 7.15 of [1]. Ultimately we show how Theorem 3 follows from results of [1].

In the following subsections of the Introduction we describe the above mentioned items in more detail. Henceforth $F$ is a free group and $H$ is a finitely generated subgroup of $F$.

\subsection{Subgroups with and without ACEP.}

$H$ falls into one of the following categories:

1. $H$ is malnormal.

2. $H$ is not cyclonormal, i.e. $\exists a \in F \backslash H \operatorname{such}$ that $\operatorname{rank}\left(H \cap H^{a}\right) \geq 2$.

3. $H$ is cyclonormal and $\exists a \in F \backslash H$ such that $H \cap H^{a}$ is generated by $u$, where $u$ is not a proper power in $F$.

4. $H$ is cyclonormal but not malnormal, and $\forall a \in F \backslash H H \cap H^{a}$ is either trivial or generated by a proper power.

Theorem 2. In the first case H, has ACEP; in the second and the third cases, $H$ does not have ACEP. In the fourth case, there are subgroups with ACEP and subgroups without ACEP.

Our proof is not difficult and uses that in the second and the third cases $H$ satisfies the property:

(S) there exists $w \in H$ such that $w^{H} \neq w^{F} \cap H$. (Here $w^{X}$ denotes the conjugacy class of $w$ in $X$.)

We see that subgroups of free groups with the property $(\mathbf{S})$ do not have ACEP. In the forth case there are subgroups with and without the property $(\mathbf{S})$. Then we continue the study of the property $(\mathbf{S})$ in Subsection 3.2, where a subgroup not having neither ACEP nor property $(\mathbf{S})$ is constructed (see Proposition 4). We also show the algorithmic decidability of the property $(\mathbf{S})$. 


\subsection{Theorem 1 and its generalization}

For $N \triangleleft H$, let $\gamma(N)=\min \{|w|, \mid w \in N \backslash\{1\}\}$. Here $|w|$ denotes the length of the reduced word, representing $w \in F$. It is clear that the following are equivalent:

- $H$ has ACEP.

- There exists $C_{H}>0$ such that $N=\langle\langle N\rangle\rangle_{F} \cap H$ whenever $\gamma(N)>C_{H}$.

Now, we are going to define $|\cdot|_{H}$, another length function on a free group $F$, to define $\gamma_{H}(N)$, and to formulate a generalization of Theorem 1 .

Definition 5. Let $F=F(X)$ be a free group on a set $X$ and $\Omega=\left\{H_{i} \mid H_{i}<F, i=1, \ldots, n\right\}$. Let $w \in F$. We define $|w|_{\Omega}=\min \left\{k \mid w=w_{1} w_{2} \cdots w_{k}, w_{i} \in H_{j}\right.$ or $\left.w_{i} \in X \cup X^{-1}\right\}$, i.e., $|w|_{\Omega}$ is the word metric with respect to a generating set $(\cup \Omega) \cup X \cup X^{-1}$.

We associate a special length function with a finitely generated $H<F$. To this end we define $\Omega(H) \subseteq$ $\left\{H^{a} \cap H^{b} \mid H a \neq H b\right\}$, put $|w|_{H}=|w|_{\Omega(H)}$ and $\gamma_{H}(N)=\min \left\{|w|_{H} \mid w \in N \backslash\{1\}\right\}$. We postpone the exact definition of $\Omega(H)$ to Subsection 4.2 but mention here two of its important properties:

1. $\Omega(H)$ is finite;

2. for each nontrivial $X \in\left\{H^{a} \cap H^{b} \mid H a \neq H b\right\}$ there is $Y \in \Omega(H)$ conjugate to $X$ in $F$.

Theorem 3. Let $H$ be an f.g.s. of $F$. There exists $C_{H}$ such that $N=\langle\langle N\rangle\rangle_{F} \cap H$ whenever $N \triangleleft H$ and $\gamma_{H}(N)>C_{H}$.

Remarks. We say that length functions $|\cdot|_{1}$ and $|\cdot|_{2}$ are Lipschitz equivalent (notation: $|\cdot|_{1} \sim|\cdot|_{2}$ ) if there are $\alpha, \beta>0$ such that $\alpha|w|_{1} \leq|w|_{2} \leq \beta|w|_{1}$ for any $w \in F$. Let $\Omega_{1}$ and $\Omega_{2}$ be finite sets of subgroups, containing the same nontrivial subgroups up to conjugacy. It is easy to check that $|\cdot| \Omega_{1} \sim|\cdot|_{\Omega_{2}}$ and that Theorem 3 holds for any length function equivalent to $|\cdot|_{H}$. So, for the statement of the theorem, the only things that matter are the properties 1) and 2) but not the exact definition of $\Omega(H)$. However, the exact definition of $\Omega(H)$ matters if we want to find $C_{H}$ constructively. Clearly, $\Omega(H) \subseteq\{1\}$ for a malnormal $H$. So, Theorem 1 is a corollary of Theorem 3. Notice that the condition " $N=\langle\langle N\rangle\rangle_{F} \cap H$ whenever $N \triangleleft H$ and $\gamma_{H}(N)>C_{H}$ " may be considered as some generalization of ACEP. It is worth mentioning that if the normalizer of $H$ is nontrivial (i.e. $\operatorname{Norm}(H) \neq H$ ) then $\Omega(H)$ contains a subgroup conjugate to $H$. So, $|\cdot|_{H}$ is bounded on $H$ and Theorem 3 is futile in this case. But it is not a weakness of the theorem. It is in the nature of things: $N=\langle\langle N\rangle\rangle_{F} \cap H$ implies that $N \triangleleft \operatorname{Norm}(H)$.

\section{Groups without ACEP}

Lemma 1. Let $F$ be a free group and $u \in F, u \neq 1$. Then $\gamma\left(\left\langle\left\langle u^{n}\right\rangle\right\rangle_{F}\right) \geq(n-1)$.

Proof. By Theorem 3 of [7] if $w \in\left\langle\left\langle u^{n}\right\rangle\right\rangle_{F}$ then $w$ contains a subword which is identical with a subword of $u^{n}$ of length greater than $(n-1) / n$ times the length of $u^{n}$. So, $\gamma\left(\left\langle\left\langle u^{n}\right\rangle\right\rangle_{F}\right) \geq(n-1)\left|u^{*}\right|$ where $u^{*}$ is a cyclic reduction of $u$.

We use the following results:

\section{Theorem 4.}

(a) Two elements $u_{1}$ and $u_{2}$ of a free group $F$ have the same normal closure in $F$ if and only if $u_{1}$ is conjugate to $u_{2}$ or to $u_{2}^{-1}$. (Magnus [8]). 
(b) In a free group F, a nontrivial commutator cannot be a proper power. (Schtzenberger [9])

(c) The solutions of equation $x^{n} y^{m}=z^{p}, n, m, p>1$ in $F$ are powers of the same element, say a. Moreover, if $x=a^{k_{x}}, y=a^{k_{y}}$, and $z=a^{k_{z}}$ then $n k_{x}+m k_{y}=p k_{z}$. (Lyndon and Schtzenberger [10]).

Let $H$ be a group, $w, a \in H$. We use the notations $w^{a}=a^{-1} w a, w^{H}=\left\{h^{-1} w h \mid h \in H\right\}$ in other words, $w^{H}$ is the conjugacy class of $w$ in $H$.

Definition 6. Let $H<F$. We say that $H$ is an $S$-subgroup of $F$ (notation: $H<_{S} F$ ) if $\exists w \in H$ such that $w^{H} \neq w^{F} \cap H$.

Proposition 1. Let $F$ be a free group. If $H<_{S} F$ then $H$ does not have ACEP in $F$.

Remark. We will see in Proposition 4 that the converse of the statement is false.

Proof. Let $w \in H$ be such that $w^{H} \neq w^{F} \cap H$. This means that $\exists a \in F \backslash H$ with $u=a^{-1} w a \in H$ such that $\forall b \in H u \neq b^{-1} w b$. Notice that $u$ cannot be conjugate to $w^{-1}$ in $H$. Otherwise, $w$ and $w^{-1}$ would be conjugate in a free group $F$, that is impossible for $w \neq 1$. By Lemma 1$] \gamma\left(\left\langle\left\langle u^{n}\right\rangle\right\rangle_{H}\right), \gamma\left(\left\langle\left\langle w^{n}\right\rangle\right\rangle_{H}\right) \geq n-1$. Now, as $u=a^{-1} w a$ we have

$$
\left\langle\left\langle u^{n}\right\rangle\right\rangle_{F}=\left\langle\left\langle w^{n}\right\rangle\right\rangle_{F} .
$$

Also $u \neq b^{-1} w b$ and $u \neq b^{-1} w^{-1} b$ for any $b \in H$. It follows (by Theorem 4 (c) with $y=1$ and $n=p$ ) that $u^{n} \neq b^{-1} w^{n} b, u^{n} \neq b^{-1} w^{-n} b$. Using once again Theorem 4 (a) we have

$$
\left\langle\left\langle u^{n}\right\rangle\right\rangle_{H} \neq\left\langle\left\langle w^{n}\right\rangle\right\rangle_{H}
$$

We deduce that $H$ does not have ACEP.

Lemma 2. Let $a \in F \backslash H$. If $\exists u=a^{-1} w a \in H \cap H^{a}$, such that $w$ is not a proper power in $F$, then $H<{ }_{S} F$.

Proof. Suppose that $\exists b \in H$ such that $u=b^{-1} w b$; then

$$
\begin{aligned}
b^{-1} w b & =u=a^{-1} w a \\
w & =\left(a b^{-1}\right)^{-1} w\left(a b^{-1}\right)
\end{aligned}
$$

therefore, $w$ commutes with $a b^{-1}$, but $F$ is a free group and this only happens if $w$ and $a b^{-1}$ are powers of the same element; by hypothesis $w$ is not a proper power, and then

$$
\begin{aligned}
a b^{-1} & =w^{n} \\
a & =w^{n} b \in H,
\end{aligned}
$$

but $a \notin H$, a contradiction.

Corollary 1. If $\operatorname{rank}\left(H \cap H^{a}\right) \geq 2$ for some $a \in F \backslash H$ then $H<_{S} F$.

Proof. Let $u, v$ be in a set of free generators of $H \cap H^{a}$. Notice that at least one element in $\{u, v, u v\}$ is not a proper power in $F$ and we are done by Lemma 2. Indeed, putting $x^{n}=u, y^{m}=v, z^{p}=u v$ implies that $\langle u, v\rangle$ is a cyclic group by Theorem 4 (c).

Corollary 2. Let $H$ contain a normal subgroup of $F$, i.e. $\exists N<H$ such that $N \triangleleft F$, then $H<{ }_{S} F$.

Proof. It follows immediately from the previous corollary.

Proof of the Theorem 2. The first case follows from Theorem 1. The second and third cases follow from Lemma 2, Corollary 1 and Proposition 1. The fourth case is studied in the next section. 


\section{The case 4}

In this section we give examples of cyclonormal subgroups $H_{1,2}$ with $H_{1,2} \cap H_{1,2}^{a}, a \notin H$, being trivial or generated by a proper power, such that $H_{1}$ does not have ACEP and $H_{2}$ has ACEP. After that we investigate the S-subgroups further and prove Proposition 4.

\subsection{Examples.}

Using Proposition 9.15 [1] we can see that the subgroups $H_{1}=\left\langle x^{n}, y^{-1} x^{n} y\right\rangle, H_{2}=\left\langle x^{n}, y^{n}\right\rangle$ are cyclonormal and every nontrivial $H_{i} \cap H_{i}^{a}, a \in F \backslash H$, is generated by a proper power. It is easy to see that $H_{1}$ does not possess ACEP while $H_{2}$ does.

To prove that $H_{2}=\left\langle x^{n}, y^{n}\right\rangle$ possesses ACEP (even CEP), let us take a group generated by 2 elements $G=\langle c, d\rangle$ and define an epimorphism $\theta: H_{2} \longrightarrow G$ such that $\theta\left(x^{n}\right)=c$ and $\theta\left(y^{n}\right)=d$. Being able to extend $\theta$ up to an epimorphism $\theta^{*}: F \longrightarrow G^{*}$ is equivalent to being able to solve the equations $x^{n}=c$, $y^{n}=d$ over $G$. But both equations have a solution according to F. Levin [12]. On the other hand, it is easy to see that $H_{1}$ does not possess ACEP. Moreover, it is an S-subgroup. One can check it directly, or, alternatively, it follows by Proposition 3 below. An example of a non-S-subgroup without ACEP is given in Proposition 4.

\subsection{S-Subgroups}

Definition 7. Let $H<_{S} F$. A pair $(w, a)$ is said to be an $S$-witness for $H$ if $w, w^{a} \in H$ and $\forall b \in H w^{a} \neq$ $w^{b}$

In this subsection we

- Show that for a finitely generated $H<F$ it is decidable whether $H<_{S} F$;

- Give an example of a finitely generated $H<F$ that is not an S-subgroup but does not possess ACEP.

Proposition 2. Let $H<F$ and $b \in F$. Then

- $H$ has $A C E P$ if and only if $H^{b}$ has $A C E P$.

- If $H<_{S} F$ with an S-witness $(w, a)$ then $H^{b}<_{S} F$ with an S-witness $\left(w^{b}, a^{b}\right)$.

We use the Stallings (folded) graphs, see [11] for details. We start with some terminology. Let $F=F(X)$ be a free group on $X$. An $X$-digraph $\Gamma$ is a directed graph with edges labeled by elements of $X$. We denote by $V . \Gamma$ and $E . \Gamma$ the set of vertices and the set of edges of $\Gamma$, respectively. For an edge $e \in E$. $\Gamma$ we denote the origin of $e$ by $o(e)$ and the terminus of $e$ by $t(e)$. The label of $e$ is denoted by $l(e)$. We introduce a formal inverse $e^{-1}$ of $e$ with label $l\left(e^{-1}\right)=l(e)^{-1}$, the origin $o\left(e^{-1}\right)=t(e)$, and terminus $t\left(e^{-1}\right)=o(e)$. (Notice that $l(e) \in X$ while $l\left(e^{-1}\right) \in X^{-1}$. We also suppose that $e^{-1} \notin E$.. . Intuitively, if one goes in the direction of an edge one reads its label, say $x$, if one goes in the opposite direction of an edge one reads the inverse of its label, say $\left.x^{-1}\right)$. A path $P$ in $\Gamma$ is a sequence $P=e_{1}, \ldots, e_{k}$ where $e_{i} \in\left(E . \Gamma \cup(E . \Gamma)^{-1}\right)$ with $o\left(e_{i}\right)=t\left(e_{i-1}\right)$ for $1<i \leq k$. A path $P$ has a naturally defined label $l(P)=l\left(e_{1}\right), \ldots, l\left(e_{k}\right)$, a naturally defined inverse path $P^{-1}=e_{k}^{-1}, \ldots, e_{1}^{-1}$ with label $l\left(P^{-1}\right)=l(P)^{-1}$. (We suppose here that $\left(e^{-1}\right)^{-1}=e$.) The origin and terminus of the path $P$ are defined as $o(P)=o\left(e_{1}\right)$ and $t(P)=t\left(e_{k}\right)$. The label of a path is just a word in the alphabet $X \cup X^{-1}$, not necessarily reduced. A path in $\Gamma$ is called reduced if it does not contains subpath $e, e^{-1}$. A cyclic path (or a cycle) is called cyclically reduced if all its cyclic permutations are reduced.

Definition 8. An $X$-digraph $\Gamma$ is called folded if for every vertex $v \in \Gamma$ and $x \in X$ there is at most one edge $e$ with $o(e)=v$ and $l(e)=x$ as well as at most one edge $e$ with $t(e)=v$ and $l(e)=x$. 


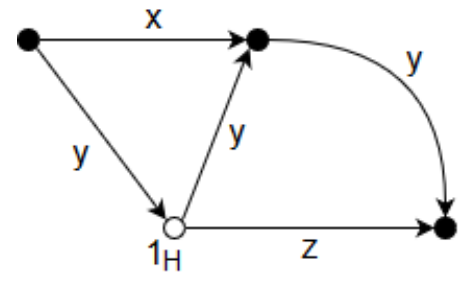

(a)

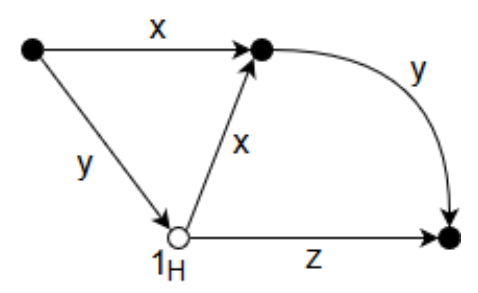

(b)

Figure 1: Folded (a) and nonfolded graphs (b).

Each (reduced) path in folded $\Gamma$ produces a (reduced) word in $X \cup X^{-1}$. We denote by $P\left(v_{1}, v_{2}, w\right)$ a path in $\Gamma$ from $v_{1}$ to $v_{2}$ with label $w$. For a folded $\Gamma, v \in V . \Gamma$, and a word $w \in\left(X \cup X^{-1}\right)^{*}$ may exist at most one path $P(v, \cdot, w)$ started from $v$ with label $w$. If $w$ is reduced, then $P(v, \cdot, w)$ is reduced. If $P(v, v, w)$ is a cycle and $w$ is cyclically reduced, then $P(v, v, w)$ is cyclically reduced. Let $H$ be an f.g.s. of a free group $F=F(X)$.

Definition 9. The Stallings graph for a subgroup $H$ is a connected folded $X$-graph $\Gamma(H)$ with a marked vertex $1_{H}$ satisfying

- $H$, as a set of reduced words, coincides with the set of labels of reduced cycles form $1_{H}$ to $1_{H}$;

- nonmarked vertex of $\Gamma(H)$ has degree at least 2.

Notice, that the marked vertex $1_{H}$ may have degree 1.

A Stallings graph $\Gamma(H)$ may be constructed using Stallings's folding, [11]. The correspondence $H \rightarrow$ $\Gamma(H)$ is a bijection, considering $\Gamma(H)$ up to isomorphisms of $X$-graphs with a marked vertex, [11]. By definition, $\Gamma=\Gamma(H)$ may be considered as a graph without edges of degree 1 , named $T y p e(\Gamma)$, with a "tail" going to $1_{H}$ attached. (The "tail" may be empty.) Formally Type $(\Gamma)$ may be constructed as follows: The only vertex of $\Gamma_{0}(H)=\Gamma(H)$ that may have degree one is $1_{H}$. In this case, removing $1_{H}$ from $V$. $\Gamma$ we obtain the induced subgraph $\Gamma_{1}(H)$. The graph $\Gamma_{1}(H)$ has at most one vertex of degree 1 . Removing it we obtain subgraph $\Gamma_{2}(H)$. Repeating this procedure, we end up with a graph $\Gamma_{k}(H)$ without vertices of degree 1. By definition, Type $(\Gamma(H))=\Gamma_{k}(H)$.

Lemma 3 ([11]). Subgroups $H_{1}$ and $H_{2}$ are conjugate in $F$ if and only if Type $\left(\Gamma\left(H_{1}\right)\right)$ and Type $\left(\Gamma\left(H_{2}\right)\right)$ are isomorphic as $X$-graphs (not respecting the marked vertices).

Proposition 3. Let $\Gamma(H)$ be the Stallings graph for $H$. Then $H<_{S} F$ if and only if there are cyclically reduced paths $P(v, v, w), P\left(v^{\prime}, v^{\prime}, w\right)$ in $\Gamma(H)$ such that one is not a cyclic permutation of the other.

Remark. The existence of such a pair of paths may be effectively checked, using, for example, the product-graph $\Gamma(H) \times \Gamma(H)$, see [11] and Subsection 4.2 .

Proof. Notice that all cyclically reduced cycles lie in Type $(\Gamma(H))$. So, by Lemma 3 and Proposition 2 , we may assume that $v^{\prime}=1_{H}$, changing $H$ by its conjugate, if necessary. All words in the proof are supposed to be cyclically reduced. So, $w_{1} w_{2}$ denotes the product in a free group, or, precisely, the corresponding reduced word.

$\Longleftarrow$ Suppose, that there are paths $P_{2}=P(v, v, w), P_{1}=P\left(1_{H}, 1_{H}, w\right)$ in $\Gamma(H)$ such that $P_{2}$ is not a cyclic permutation of $P\left(1_{H}, 1_{H}, w\right)$. There is a reduced path $Q$ from $v$ to $1_{H}$. Let $a=l(Q)$. We show that $(w, a)$ is an S-witness for $H$. First of all, $a \notin H$ and the reduction of $Q^{-1} P_{2} Q$ is $P\left(1_{H}, 1_{H}, a^{-1} w a\right)$. So, $w, a^{-1} w a \in H$. Suppose, searching for a contradiction, that $(w, a)$ is not an S-witness. Then there exists $b \in H$, such that $a^{-1} w a=b^{-1} w b$. The reduction of $P\left(1_{H}, 1_{H}, b\right) P\left(1_{H}, v, a^{-1}\right)$ is a path $P\left(1_{H}, v, u\right)$ with $u=$ $b a^{-1} \neq 1$. As $[u, w]=1$ we have $w=y^{n}$ and $u=y^{m}$. Since $w$ is cyclically reduced, $y$ is cyclically reduced 
as well. So, product $y y$ is just a concatenation (no cancellations happen). $P\left(1_{H}, v, u=y^{m}\right.$ ) is a subpath of $P\left(1_{H}, 1_{H}, w=y^{n}\right)$ or vice versa (by uniqueness of paths with given label and origin in folded graphs). In either case, we deduce that $P\left(1_{H}, 1_{H}, w\right)=P\left(1_{H}, v, y^{k}\right) P\left(v, 1_{H}, y^{n-k}\right)$ and $P_{2}=P\left(v, 1_{H}, y^{n-k}\right) P\left(1_{H}, v, y^{k}\right)$ is a cyclic permutation of $P_{1}$, a contradiction.

$\Longrightarrow$ Suppose that $H<_{S} F$ and $(w, a)$ is an S-witness. W.l.g. we assume that $w$ is cyclically reduced. It implies that either $a^{-1} w$ or $w a$ is a product without cancellation. Changing, if necessary, $w \rightarrow w^{-1}$, w.l.g. we may assume that $a^{-1} w$ is the product without cancellation. We may assume as well that not all letters of $w$ are canceled in $w a$. (If not, then change $a \rightarrow w a$. It may happen that after this we have to change $w \rightarrow w^{-1}$, and, if necessary, repeat the process several times.) As $a^{-1} w a \in H$ and the product of $a^{-1}$ and $w a$ is without cancellations paths $P\left(1_{H}, 1_{H}, a^{-1} w a\right)=P\left(1_{H}, v, a^{-1}\right) P\left(v, 1_{H}, w a\right)$ exist. Now, there is a path $P(v, v, w)$ which is the reduction of $P\left(v, 1_{H}, w a\right) P\left(1_{H}, v, a^{-1}\right)$. So, there are $P_{1}=P\left(1_{H}, 1_{H}, w\right)$ and $P_{2}=P(v, v, w)$. Suppose, searching for a contradiction, that $P_{2}$ is a cyclic permutation of $P_{1}$. In other words, $P_{1}=P\left(1_{H}, v, y\right) P\left(v, 1_{H}, z\right)$ and $P_{2}=P\left(v, 1_{H}, z\right) P\left(1_{H}, v, y\right)$. So, $w=y z=z y$. It implies that $y^{-1} w y=w, b=y a \in H$ and $b^{-1} w b=a^{-1} w a$, a contradiction.

Proposition 4. Let $H=\left\langle a^{4}, a^{2} b a, a c a^{2}, b c^{-1}\right\rangle$. Then $H$ is not an $S$-subgroup of $F(a, b, c)$ and does not have ACEP.

Proof. First of all, using the Stallings graph $\Gamma(H)$ and Lemma 6.1 [1] we see that $H$ is freely generated by

$$
w_{1}=a^{4}, w_{2}=a^{2} b a, w_{3}=a c a^{2}, w_{4}=b c^{-1} .
$$

Let $N=\left\langle\left\langle\left[w_{1}^{n}, w_{2}\right],\left[w_{1}^{n}, w_{3}\right]\right\rangle\right\rangle_{H}, n \in \mathbb{Z}$ and $[\cdot, \cdot]$ is a commutator. One can check that $\gamma(N) \geq\left\lfloor\frac{n-1}{2}\right\rfloor$ and $\left[w_{1}^{n}, w_{4}\right] \notin N$. Indeed,

$$
H / N=\left(\left\langle u, w_{2}, w_{3} \mid\left[u, w_{2}\right],\left[u, w_{3}\right]\right\rangle \underset{u=w_{1}^{n}}{*}\left\langle w_{1}\right\rangle\right) *\left\langle w_{4}\right\rangle,
$$

Consider the image of $\left\langle w_{1}, w_{2}, w_{3}\right\rangle$ in $H / N$. The elements of $H / N$ have the following normal form:

$$
w_{1}^{r n} p_{1}\left(w_{2}, w_{3}\right) w_{1}^{\alpha_{1}} \ldots
$$

where $p_{i} \in\left\langle w_{2}, w_{3}\right\rangle$ and $-\left\lfloor\frac{n-1}{2}\right\rfloor \leq \alpha \leq\left\lfloor\frac{n}{2}\right\rfloor$, see [13]. It follows that any word in $w_{1}, \ldots, w_{4}$ of length less than $\left\lfloor\frac{n-1}{2}\right\rfloor$ is in the normal form and does not belong to $N$. Also, it is easy to check that $\left[w_{1}^{n}, w_{4}\right]=$ $w_{1}^{n} w_{4} w_{1}^{-n} w_{4}^{-1} \notin N$. But $\left[w_{1}^{n}, w_{4}\right] \in\langle\langle N\rangle\rangle_{F}$. Indeed, $w_{4}=a^{-2} w_{2} a w_{3}^{-1} a$ and $\left[w_{1}, a\right]=1$ by definitions of $w_{i}$.

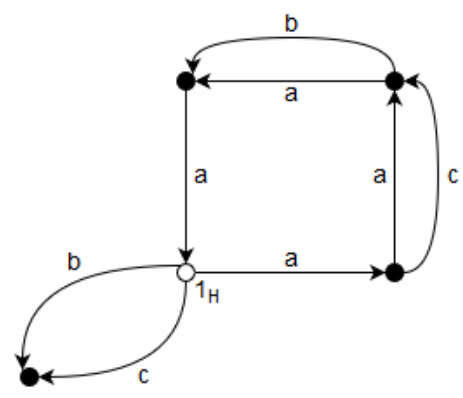

Figure 2: $\Gamma(H)$

Using the graph $\Gamma(H)$ we can see that there are no cyclically reduced paths $P(v, v, h), P\left(v^{\prime}, v^{\prime}, h\right) \in \Gamma(H)$ such that $P(v, v, h)$ is not a cyclic permutation of $P\left(v^{\prime}, v^{\prime}, h\right)$. Then by Proposition $3 H$ is not an $S$ subgroup. 


\section{Proof of Theorem 3}

In this section we prove Theorem 1 by means of the small cancellation theory (see [8]) with the use of Stallings's folded graphs. Let $H$ be an f.g.s. of a free group $F$ and $N \triangleleft H$. We start with the construction of derivation diagrams for $u \in\langle\langle N\rangle\rangle_{F}$.

\subsection{Derivation diagram for $u \in\langle\langle N\rangle\rangle_{F}$}

Definition 10. A morphism from an $X$-digraph $\Gamma_{1}$ to an $X$-graph $\Gamma_{2}$ is a map $\pi$ from the set of vertices and edges of $\Gamma_{1}$ to the set of vertices and edges of $\Gamma_{2}$ sending vertices to vertices, edges to edges, preserving labels $(l(e)=l(\pi(e)))$ and extremes of edges $(o(\pi(e))=\pi(o(e)), t(\pi(e))=\pi(t(e)))$.

Definition 11. Let E. $\Gamma$ be the set of edges of $\Gamma$. The in-star of a vertex $v$ in a graph $\Gamma$ is the set instar $(v)=$ $\{e \in E . \Gamma \mid v=t(e)\}$. The out-star of a vertex $v$ in a graph $\Gamma$ is the set outstar $(v)=\{e \in E . \Gamma \mid v=o(e)\}$

Definition 12. A graph $\Gamma_{1}$ is a covering graph of a graph $\Gamma_{2}$ if there is a covering map from $\Gamma_{1}$ to $\Gamma_{2}$. A covering map $f: \Gamma_{1} \rightarrow \Gamma_{2}$ is an X-digraph epimorphism such that the in-star and out-star of each vertex $v$ of $\Gamma_{1}$ is mapped bijectively onto the in-star and out-star of its image $f(v)$, respectively.

Notice that a covering graph for a folded graph is folded.

Proposition 5. Let $H<F, N \triangleleft H$, and $\Gamma(H)$ be the corresponding Stallings folded graph for $H$. There is a connected $X$-digraph $\Gamma(N)$ that is a covering of $\Gamma(H)$ with a covering map $f: \Gamma(N) \rightarrow \Gamma(H)$ satisfying the following property:

*) for every $v \in f^{-1}\left(1_{H}\right)$ the group $N$ (as a set of reduced words) coincides with the labels of reduced paths from $v$ to $v$.

Property *) uniquely (up to isomorphism) determines a connected covering of $\Gamma(H)$.

Proof. We give a precise construction for $\Gamma(N)$ as the covering graph of $\Gamma(H)$ with a desk transformation $H / N$. Fix a spanning tree $T$ in $\Gamma(H)$. For $v \in V . \Gamma(H)$ let $P(v)$ be the reduced path in $T$ from $1_{H}$ to $v$. For each edge $e \notin E . T$ let $h_{e}$ be the label of the path $P(o(e)) e P^{-1}(t(e))$. The set $\left\{h_{e} \mid e \in E . \Gamma(H) \backslash E . T\right\}$ generates $H$ freely, [11]. Let $G=H / N$ and $\bar{h}$ denote the image of $h \in H$ in $G$ by the natural homomorphism $H \rightarrow H / N$. We define $\Gamma(N)$ as follows:

- $V . \Gamma(N)=V . \Gamma(H) \times G$,

- $E . \Gamma(N)=E . \Gamma(H) \times G$,

- $l(e, g)=l(e), o(e, g)=(o(e), g)$, and $t(e, g)= \begin{cases}(t(e), g), & \text { if } \quad e \in T \\ \left(t(e), g \bar{h}_{e}\right) & \text { if } \quad e \notin T\end{cases}$

It is routine to check that $N$ coincides with labels of the reduced cycles of $\left(1_{H}, g\right)$ of $\Gamma(N)$ and that map $f: \Gamma(N) \rightarrow \Gamma(H), f(a, g)=a$, is a covering map. Notice that it is not difficult to construct an isomorphism between two connected covering graphs of $\Gamma(H)$ with property $\left.{ }^{*}\right)$.

Let $f: \Gamma_{1} \rightarrow \Gamma_{2}$ be an $X$-digraph morphism. We naturally extend $f$ to the inverses of edges: $f\left(e^{-1}\right)=$ $(f(e))^{-1}$. For any path $Q=e_{1}, e_{2}, \ldots, e_{k}$ in $\Gamma_{1}$ its image $f(Q)=f\left(e_{1}\right), f\left(e_{2}\right), \ldots, f\left(e_{k}\right)$ is a path in $\Gamma_{2}$. It is clear by definition that $l(Q)=l(f(Q))$. If $P$ is a path in $\Gamma_{2}$, a lift of $P$ is a path $Q$ in $\Gamma_{1}$ such that $f(Q)=P$.

Lemma 4. Let $\Gamma_{1}, \Gamma_{2}$ be graphs and $f: \Gamma_{1} \longrightarrow \Gamma_{2}$ be a covering map. If $P$ is a path in $\Gamma_{2}$ then for any $v \in f^{-1}(o(P))$, there is a unique lift of $P$ starting at $v$. 
Lemma 5. Let $N \triangleleft H$ and $f: \Gamma(N) \longrightarrow \Gamma(H)$ be a covering map. Then $\forall v \in V . \Gamma(H)$ and $\forall v_{1}, v_{2} \in$ $f^{-1}(v)$ there exist an automorphism $\alpha: \Gamma(N) \longrightarrow \Gamma(N)$ such that $\alpha\left(v_{1}\right)=v_{2}$.

Proof. Indeed, $v_{1}=\left(v, h_{1}\right), v_{2}=\left(v, h_{2}\right)$, and $\alpha(a, h)=\left(a,\left(h_{2} h_{1}^{-1}\right) h\right)$.

A presentation $\langle X \mid R\rangle$ is called symmetrized if $R$ consists of cyclically reduced words and for any $w \in R$ all cyclic permutations of $w$ as well as $w^{-1}$ belong to $R$. A diagram $M$ over the symmetrized presentation $\langle X \mid R\rangle$ is a planar finite cell complex (which we denote, abusing notation, by $M$ as well), given with a specific embedding $M \subseteq \mathbb{R}^{2}$, the following additional data, and satisfying the following additional properties:

1. The complex $M$ is connected and simply connected.

2. Each edge (one-cell) of $M$ is labeled by an arrow and a letter $x \in X$.

3. Some vertex (zero-cell) which belongs to the topological boundary $\delta M$ of $M \subseteq \mathbb{R}^{2}$ is specified as a base-vertex.

4. For each region (two-cell) $D$ of $M$ the label of boundary cycle is in $R$, (in our notations $l(\delta(D)) \in R)$. (Notice that $l(\delta(D))$ is defined up to cyclic permutation, if we do not specify a base vertex of $\delta(D)$.)

Thus the 1-skeleton of $M$ is a finite connected planar graph $M^{1}$ embedded in $\mathbb{R}^{2}$ and the two-cells of $M$ are precisely the bounded complementary regions for this graph. Each geometric edge $e$ has two orientations. We chose the positive orientation, such that the label of $e$ is in $X$. With this convention we consider $M^{1}$ as an $X$-digraph with $E . M^{1}$ being a set of positively oriented edges. For a two-cell $D$ its boundary $\delta(D)$ is a closed path $e_{1}, v_{1}, e_{2}, v_{2} \ldots e_{k} v_{k}$ in $M^{1}$ with edges $e_{j} \in E . M^{1} \cup\left(E . M^{1}\right)^{-1}$ and vertices $v_{j} \in V \cdot M^{1}$. A diagram $M$ also has the boundary cycle, denoted by $\delta M$, which is an edge-path in the graph $M^{1}$ corresponding to going around $M$ once in the clockwise direction along the boundary of the unbounded complementary region of $M^{1}$, starting and ending at the base-vertex of $M$. The label of that boundary cycle is a word $w$ in the alphabet $X \cup X^{-1}$ that is called the boundary label of $M$. We call $M$ a derivation diagram for $w$. It is a lemma of Van Kampen that $w \in\langle\langle R\rangle\rangle_{F}$ if and only if a derivation diagram for $w$ exist, see [8] page 237. As we are interested in $\langle\langle N\rangle\rangle_{F}$ for $N \triangleleft H<F$ we chose $R$ to be the set of labels of all cyclically reduced cycles in $\Gamma(N)$. This implies that for any region $D \in M$ there is a cycle $C_{D}=P(v, v, w)$ in $\Gamma(N)$ with $w=l(\delta(D))$. It is possible that a vertex of $M^{1}$ appears more than once in $\delta(D)$. We call such a vertex a multiple vertex of $\delta(D)$. Also, it is possible that both $e$ and $e^{-1}$ are in $\delta(D)$. We call such an edge a double edge of $\delta(D)$. A graph formed by multiple vertices and double edges of $\delta(D)$ is called the self-boundary of $D$. Notice that a connected component of a self-boundary is a tree. Let $\delta(D)=e_{1}, v_{1}, e_{2}, v_{2}, \ldots, e_{k}, v_{k}$ with edges $e_{i}$ and vertices $v_{i}$. Changing $\delta(D)$ by $\tilde{\delta}(D)=\left(1, e_{1}\right),\left(1, v_{1}\right),\left(2, e_{2}\right),\left(2, v_{2}\right), \ldots,\left(k, e_{k}\right),\left(k, v_{k}\right)$, we get a cycle with different vertices and edges with labels $l\left(\left(j, e_{j}\right)\right)=l\left(e_{j}\right)$. Intuitively, we think of $\tilde{\delta}(D)$ as an infinitesimal shift of $\delta(D)$ inside of $D$. In $\tilde{\delta}(D)$ there are edges with labels from $X$ (positive edges) and edges with labels from $X^{-1}$ (negative edges). Changing the negative edges in $\tilde{\delta}(D)$ by its inverse we obtain an $X$-digraph $\delta^{\prime}(D)$. By construction there exists a morphism $\phi_{D}: \delta^{\prime}(D) \rightarrow \Gamma(N)$.

Definition 13. Let $w \in\langle\langle N\rangle\rangle_{F}$. A derivation $N$-diagram for $w$ is a diagram $M$ with the following properties:

- $l(\delta(M))=w$, where $\delta(M)$ denotes the boundary of $M$.

- For each label of an internal region of $M$ there exists a cyclically reduced cycle in $\Gamma(N)$ with the same label. So, for a region $D$ there exists a labeled graph morphism $\phi_{D}: \delta^{\prime}(D) \rightarrow \Gamma(N)$. 
The discussion above shows that $w \in\langle\langle N\rangle\rangle_{F}$ iff a derivation $N$-diagram for $w$ exist. We call a path $P$ in $M^{1}$ a piece if degrees of $o(P)$ and $t(P)$ are at least 3 and degrees of all other vertices of $P$ are 2. We call a piece $P$ external if $P \in \delta(M)$, otherwise we call it internal. In what follows we consider internal pieces only, so "piece" means "internal piece" through the text. Let $P$ be a piece. There exist regions $D$ and $E$ such that $P \in \delta(D)$ and $P^{-1} \in \delta(E)$. There is a natural image $P_{D}$ (resp. $\left.P_{E}\right)$ of $P$ in $\delta^{\prime}(D)$ (resp. $\delta^{\prime}(E)$ ), see fig. 3. If $E=D$ then $P$ is a path in the self-boundary of $D$. In this case, there are two different paths in $\delta^{\prime}(D)$ corresponding to $P$. We say that a piece $P$ is inessential if there are morphisms $\phi_{D}: \delta^{\prime}(D) \rightarrow \Gamma(N)$ and $\phi_{E}: \delta^{\prime}(E) \rightarrow \Gamma(N)$ such that $f\left(\phi_{D}\left(P_{D}\right)\right)=f\left(\phi_{E}\left(P_{E}\right)\right)$. (Recall that $f: \Gamma(N) \rightarrow \Gamma(H)$ is a covering map. Also, if $D=E$ we assume that $\phi_{D}=\phi_{E}$.) We say that $P$ is essential if it is not inessential. For an $N$-diagram $M$ let $r(M)$ be a number of regions. For $u \in\langle\langle N\rangle\rangle_{F}$ let $r(u)=\min \{r(M) \mid M$ is a derivation $N$-diagram for $u\}$. A derivation $N$-diagram $M$ for $u$ is called optimal if $r(M)=r(u)$.

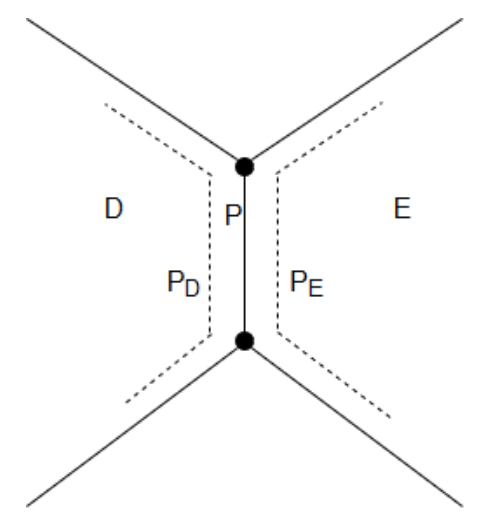

Figure 3: The piece $\mathrm{P}$ in the $\mathrm{D}$ and $\mathrm{E}$ regions

Lemma 6. An inessential piece of an optimal $N$-diagram is a path in a self-boundary of a region.

Proof. Let $M$ be a derivation $N$-diagram for $u$. Suppose, that a piece $P \subseteq \delta(D) \cap \delta(E)$ is inessential and $D \neq E$. We may write $\delta(D)=Q_{1} P$ and $\delta(E)=P^{-1} Q_{2}$. Let $D^{\prime}$ be a region, obtained by gluing $D$ and $E$ along $P$. Construct $M^{\prime}$ by removing $D \cup E$ and gluing $D^{\prime}$ along $Q_{1} Q_{2}$. If $Q_{1} Q_{2}$ is not cyclically reduced we may apply folding to $M^{\prime}$, see section 5.1 of [8]. Clearly, the resulting diagram has $r(M)-1$ region. To get a contradiction it suffices to show that it is an $N$-diagram or, the same, that in $\Gamma(N)$ there exists a cycle with label $l\left(Q_{1} Q_{2}\right)$. But $f\left(\phi_{D}(P)\right)=f\left(\phi_{E}(P)\right)$ for some $\phi_{D}, \phi_{E}$. By Lemma 5 there is an automorphism $\alpha: \Gamma(N) \rightarrow \Gamma(N)$ such that $\alpha\left(\phi_{D}(P)\right)=\phi_{E}(P)$. So, $\alpha\left(\phi_{D}\left(Q_{1}\right)\right) \phi_{E}\left(Q_{2}\right)$ is a required cycle .

Let $P$ be an inessential piece in the self-boundary of $D$, i.e. $\delta(D)=Q_{1} P Q_{2} P^{-1}$. $P$ splits in $\delta^{\prime}(D)$ in two paths, say $P_{1}$ and $P_{2}$. It may happen that $\phi_{D}\left(P_{1}\right) \neq \phi_{D}\left(P_{2}\right)$ for a morphism $\phi_{D}: \delta^{\prime}(D) \rightarrow$ $\Gamma(N)$. By definition of an inessential boundary component $f\left(\phi_{D}\left(P_{1}\right)=f\left(\phi_{D}\left(P_{2}\right)\right.\right.$ for some morphism $\phi_{D}$. (Particularly, it implies that $f\left(\phi_{D}\left(Q_{i}\right)\right)$ are cycles in $\Gamma(H)$.)

\subsection{Length functions}

In this subsection we give a detailed definition of $|\cdot|_{H}$. Recall the definition of $|\cdot|_{\Omega}$ in Subsection 1.2 . Recall, as well, that $|\cdot|_{H}=|\cdot|_{\Omega(H)}$. So, the only thing we need is a detailed definition of $\Omega(H)$. We start with some properties of $\Gamma(H)$.

Proposition 6. Let $v_{i} \in \Gamma(H)$ and $P\left(1_{H}, v_{i}, w_{i}\right)$ be reduced paths in $\Gamma(H)$ for some $w_{i} \in F, i=1,2$. Then $w_{1} w_{2}^{-1} \in H$ if and only if $v_{1}=v_{2}$.

Proof. The proof uses the uniqueness in $\Gamma(H)$ of paths with given origin and label. 
$\Longrightarrow$ Let $w_{1}=w_{1}^{\prime} \alpha$ and $w_{2}=w_{2}^{\prime} \alpha$ be such that $w_{1}^{\prime}\left(w_{2}^{\prime}\right)^{-1}$ and $w_{i}^{\prime} \alpha$ are products without cancellation. As $w_{1}^{\prime}\left(w_{2}^{\prime}\right)^{-1}=w_{1} w_{2}^{-1} \in H$, there is a cycle $P\left(1_{H}, 1_{H}, w_{1}^{\prime} w_{2}^{\prime-1}\right)=P\left(1_{H}, v^{\prime}, w_{1}^{\prime}\right) P\left(v^{\prime}, 1_{H}, w_{2}^{\prime-1}\right)$. Now,

$$
P\left(1_{H}, v_{1}, w_{1}\right)=P\left(1_{H}, v^{\prime}, w_{1}^{\prime}\right) P\left(v^{\prime}, v_{1}, \alpha\right) \text { and } P\left(1_{H}, v_{2}, w_{2}\right)=P\left(1_{H}, v^{\prime}, w_{2}^{\prime}\right) P\left(v^{\prime}, v_{2}, \alpha\right)
$$

So, $v_{1}=v_{2}$ by the uniqueness of the path $P\left(v^{\prime}, \cdot, \alpha\right)$.

$\Longleftarrow$ Similar.

Consider a path $P\left(1_{H}, v, w^{-1}\right)$ in $\Gamma(H)$. By Proposition 6, $H^{w}$ depends on $v$ only. So, for $v \in V$. $\Gamma(H)$ we define $H^{v}=H^{w}$ for some $w \in F$ with $P\left(1_{H}, v, w^{-1}\right)$. Actually, $H^{v}$ consists of labels of the reduced cycles from $v$ to $v$ in $\Gamma(H)$. Now, let $\Omega(H)=\left\{H^{a} \cap H^{b} \mid a, b \in V . \Gamma(H) \wedge a \neq b\right\}$. We define $|w|_{H}=|w|_{\Omega(H)}$.

Definition 14. We define $\operatorname{diam}(\Gamma)=\max \{d(u, v) \mid u, v \in V . \Gamma, d(u, v)<\infty\}$, where $d(u, v)$ is a graph distance, that is, the length of the shortest path from $u$ to $v$. If $P$ is a path in $\Gamma$, we denote by $|P|$ the length of $P$. Notice that diam $(\Gamma)$ is finite for a finite graph $\Gamma$ (even disconnected).

Consider $\Gamma(H) \times \Gamma(H)$, the product of $\Gamma(H)$ in the category of $X$-labeled graphs. That is, $V .(\Gamma \times \Gamma)=$ $(V . \Gamma) \times(V . \Gamma)$ and $l_{\Gamma \times \Gamma}^{-1}(x)=l_{\Gamma}^{-1}(x) \times l_{\Gamma}^{-1}(x)$. Where $l_{G}^{-1}(x)$ is the set of edges with label $x$ in a graph $G$. Notice that path $P\left(\left(v_{1}, v_{1}^{\prime}\right),\left(v_{2}, v_{2}^{\prime}\right), w\right)$ in $\Gamma \times \Gamma$ defines two paths $P\left(v_{1}, v_{2}, w\right)$ and $P\left(v_{1}^{\prime}, v_{2}^{\prime}, w\right)$ in $\Gamma$. It follows that $H^{v} \cap H^{u}$ consists of the labels of cycles from $(v, u)$ to $(v, u)$ in $\Gamma(H) \times \Gamma(H)$. The next proposition follows from the properties of folded graphs.

Proposition 7. Suppose, that there is an edge from $(u, v)$ to $\left(u^{\prime}, v^{\prime}\right)$ in $\Gamma(H) \times \Gamma(H)$. Then $u=v$ if and only if $u^{\prime}=v^{\prime}$.

So, $\Gamma(H) \times \Gamma(H)$ contains a diagonal connected component isomorphic to $\Gamma(H)$. Let $\Gamma(H) \dot{\times} \Gamma(H)$ be $\Gamma(H) \times \Gamma(H)$ with the diagonal component removed. Let $C=\operatorname{diam}(\Gamma(H) \dot{\times} \Gamma(H))+1$. (Notice that $C$ is finite by Definition 14 .)

Lemma 7. Let $w \in F$ and suppose that there exists a path $P(v, u, w)$ in $\Gamma(H) \dot{\times} \Gamma(H)$. Then $|w|_{H} \leq C$

Proof. Take $a \in F,|a|<C$, such that there is a path $P(u, v, a)$ in $\Gamma(H) \dot{\times} \Gamma(H)$. It follows that $P(v, v, w a)$ exist. So, $w a \in H^{v_{1}} \cap H^{v_{2}} \in \Omega(H)$ where $v=\left(v_{1}, v_{2}\right), v_{1} \neq v_{2}$. Now, $|w|_{H}=\left|w_{a a^{-1}}\right|_{H} \leq 1+|a| \leq C$.

Corollary 3. Let $w \in F,|w|_{H}>C$. There exists at most one path with label $w$ in $\Gamma(H)$.

Corollary 4. Let $N \triangleleft H$ and $M$ be an $N$-diagram. Let $P$ be an essential piece in $M$. Then $|l(P)|_{H} \leq C$.

Proof. $f\left(\phi_{D}(P)\right) \neq f\left(\phi_{E}(P)\right)$ for all $\phi_{D}, \phi_{E}$ of Definition 13. Fix such $\phi_{D}, \phi_{E}\left(\phi_{D}=\phi_{E}\right.$ if $\left.D=E\right)$. It follows that in $\Gamma(H)$ there are two different paths with label $l(P)$. They define a path in $\Gamma(H) \dot{\times} \Gamma(H)$ and the proof is concluded by Lemma 7 .

Lemma 8. Let $N \triangleleft H$. If a path $P(v, v, w)$ in $\Gamma(N)$ is cyclically reduced then $|w|_{H} \geq \gamma_{H}(N)-$ $2 \operatorname{diam}(\Gamma(H))$.

Proof. Let $f: \Gamma(N) \rightarrow \Gamma(H)$ be a covering map, $u=f(v)$. There is a path $P\left(1_{H}, u, b\right)$ in $\Gamma(H)$ with $|b|_{H} \leq \operatorname{diam}(\Gamma(H))$. Let $Q$ be the lift of $P\left(1_{H}, u, b\right)$ with $t(Q)=v$. The cycle $Q P_{w} Q^{-1}$ shows that bwb $b^{-1} \in N$, but $\gamma_{H}(N) \leq\left|b w b^{-1}\right|_{H} \leq|w|_{H}+2|b|$.

\subsection{Proof of Theorem 3}

Here we prove a version of Theorem 3 with an explicit estimate.

Theorem. Let $N \triangleleft H, C=\operatorname{diam}(\Gamma(H) \dot{\times} \Gamma(H))+1$ and $\gamma_{H}(N) \geq 6 C+2 \operatorname{diam}(\Gamma(H))$. Then $N=$ $\langle\langle N\rangle\rangle_{F} \cap H$. 
Let $\Sigma=\left(\langle\langle N\rangle\rangle_{F} \cap H\right) \backslash N$. In order to prove the theorem it suffices to show that $\Sigma=\emptyset$ under conditions of the theorem.

Lemma 9. Let $N \triangleleft H$ and $\Sigma=\left(\langle\langle N\rangle\rangle_{F} \cap H\right) \backslash N$. If $w \in \Sigma, \tau \in N$ and $h \in H$ then $\tau w \in \Sigma$ and $w^{h} \in \Sigma$.

Proof. As $N<\langle\langle N\rangle\rangle_{F}$ and $N \triangleleft H, \tau w \in\langle\langle N\rangle\rangle_{F}$ and $\tau w \in H$. Suppose that $\tau w \in N$, then $w \in \tau^{-1} N=$ $N$, but $w \notin N$, it's a contradiction. For the second part, it's clear that $w^{h} \in\langle\langle N\rangle\rangle_{F} \cap H$. Suppose $w^{h} \in N$, then $w \in h N h^{-1}=N$, but $w \notin N$, it's a contradiction.

Proposition 8. Let $w_{1}, w_{2} \in F$ and $w_{1}$ be cyclically reduced. Suppose, that $w_{1} w_{2}=w_{2} w_{1}$ and there is a cycle $P\left(v, v, w_{1}\right)$ in $\Gamma(H)$. Then a path $P\left(v, v^{\prime}, w_{2}\right)$ and a cycle $P\left(v^{\prime}, v^{\prime}, w_{1}\right)$ exist in $\Gamma(H)$.

Proof. Notice that $w_{1}$ and $w_{2}$ are powers of the same element. The result follows by uniqueness of a path with a given origin and label in a folded graph.

Lemma 10. Let $a w^{*} a^{-1} \in H$ and $w^{*}$ be cyclically reduced. Then a path $P\left(1_{H}, v, a\right)$ and a cycle $P\left(v, v, w^{*}\right)$ exist in $\Gamma(H)$.

Proof. If the products in $a w^{*} a^{-1}$ are without cancellation, then the conclusion of the lemma is valid by definition of $\Gamma(H)$. Otherwise, there is a presentation $w^{*}=\alpha_{1} \alpha_{2}$ and $a w^{*} a^{-1}=a^{\prime} \alpha_{2} \alpha_{1} a^{-1}$ such that all products in $\alpha_{1} \alpha_{2}$ and $a^{\prime} \alpha_{2} \alpha_{1} a^{\prime-1}$ are without cancellation. Then there are the following paths in $\Gamma(H)$ :

$$
P\left(1_{H}, 1_{H}, a^{\prime} \alpha_{2} \alpha_{1} a^{\prime-1}\right)=P\left(1_{H}, v^{\prime}, a^{\prime}\right) P\left(v^{\prime}, v, \alpha_{2}\right) P\left(v, v^{\prime}, \alpha_{1}\right) P^{-1}\left(1_{H}, v^{\prime}, a^{\prime}\right) .
$$

So, there is a path $P\left(1_{H}, v, a^{\prime} \alpha_{1}^{-1}\right)$ as a reduction of $P\left(1_{H}, v^{\prime}, a^{\prime}\right) P^{-1}\left(v, v^{\prime}, \alpha_{1}\right)$ and a path $P\left(v, v, w^{*}\right)=$ $P\left(v, v^{\prime}, \alpha_{1}\right) P\left(v^{\prime}, v, \alpha_{2}\right)$. Notice that $a=a^{\prime} \alpha_{1}^{-1} \beta$ where $\beta \in F$ commute with $w^{*}$. The lemma follows by Proposition 8 .

Suppose that $\Sigma \neq \emptyset$. Choose $u \in \Sigma$ with $r(u)=\min \{r(y) \mid y \in \Sigma\}$ and an optimal derivation $N$-diagram $M$ for $u$. If $r(u)=1$ then $u=a w a^{-1} \in H$, with $u \in H, w \in N$ and $a \in F \backslash H$. One can write $w=x w^{*} x^{-1}$ and $u=y w^{*} y^{-1}$ with a cyclically reduced $w^{*}$. By Lemma 10 , there are paths $P\left(1_{H}, v_{x}, x\right), P\left(1_{H}, v_{y}, y\right)$ and cycles $P\left(v_{x}, v_{x}, w^{*}\right), P\left(v_{y}, v_{y}, w^{*}\right)$. Moreover, $v_{x} \neq v_{y}$ by Proposition 6. It follows that $w^{*} \in H^{v_{x}} \cap H^{v_{y}}$ and $\left|w^{*}\right|_{H}=1$. Take $z \in F,|z| \leq \operatorname{diam}(\Gamma(H))$, such that a path $P\left(1_{H}, v_{x}, z\right)$ exist in $\Gamma(H)$. Then $z x^{-1} \in H$ and $w^{\prime}=z w^{*} z^{-1} \in N$. But $\left|w^{\prime}\right|_{H} \leq 1+2|z|<\gamma(N)$ would be a contradiction.

Therefore, suppose that $r(u) \geq 2$ and $M$ has more than one region. There are two possibilities:

1. All pieces are essential.

2. There is a inessential piece.

In the first case, by Lemma 8 and Corollary 4 the number of pieces in $\delta(D)$ for a region $D$ is, at least, 6 if $\delta(D) \cap \delta(M)$ does not contain an edge. So, $M$ is a (3,6)-diagram, [8], (chapter 5). Theorem 4.3 of [8] is applicable because, for any region $D$, its boundary $\delta(D)$ does not contain vertices of degree 1 . It implies that there is a region $D$ of $M$ such that $\delta(M) \cap \delta(D)$ contains a subpath $Q$ of $\delta(D)$ with

$$
|l(Q)|_{H} \geq 3 C>C
$$

Let $v_{0} \in \delta(M)$ be a base vertex of $\delta(M)$ for $u$. Let $v_{1}=o(Q), v_{2}=t(Q)$. Let $P_{D}$ be a path in $\delta(D)$, such that $Q P_{D}$ is a cycle $\delta(D)$. Let $P_{1}$ and $P_{2}$ be paths in $\delta(M)$ such that $o\left(P_{1}\right)=v_{0}, t\left(P_{1}\right)=v_{1}$, $o\left(P_{2}\right)=v_{2}, t\left(P_{2}\right)=v_{0}$ and $u=l\left(P_{1}\right) l(Q) l\left(P_{2}\right)$. Denote $l\left(P_{1}\right)=x_{1}, l\left(P_{2}\right)=x_{2}, l(Q)=y, l\left(P_{D}\right)=z$. In $\Gamma(N)$ there is a cycle $A$ with the label $y z$, its image $f(A)$ is a cycle in $\Gamma(H)$ with the same label. Also in $\Gamma(H)$ there are paths $P\left(1_{H}, \tilde{v}_{1}, x_{1}\right), P\left(\tilde{v}_{1}, \tilde{v}_{2}, y\right)$ and $P\left(\tilde{v}_{2}, 1_{H}, x_{2}\right)$, see figure 4 . A path in $\Gamma(H)$ with label $y$ is unique by Corollary 3 . So, $P\left(\tilde{v}_{1}, \tilde{v}_{2}, y\right)$ is a subpath of $f(A)$. It follows that the path $P\left(1_{H}, \tilde{v}_{1}, x_{1}\right)$ has a lift $B$ on $\Gamma(N)$ such that $B A B^{-1}$ is a cycle in $\Gamma(N)$. So, $x_{1} y z x_{1}^{-1} \in N$ by Proposition 5 and 
$u^{\prime}=x_{1} z^{-1} x_{2}=\left(x_{1} z^{-1} y^{-1} x_{1}^{-1}\right)\left(x_{1} y x_{2}\right) \in \Sigma$ by Lemma 9. But $u^{\prime}$ has a derivation $N$-diagram with $r(u)-1$ regions. This contradiction with minimality of $r(u)$ refutes the first case.

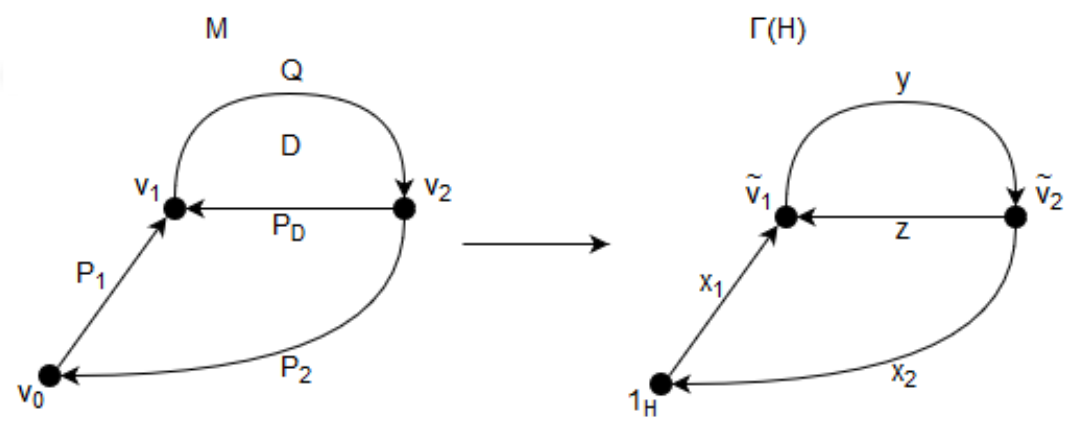

Figure 4: $M$ and $\Gamma(H)$

Consider the second case. By Lemma 6 an inessential piece $P$ is a path in the self-boundary of a region $D$. So, $\delta(D)=Q_{1} P Q_{2} P^{-1}$. $Q_{1}$ and $Q_{2}$ define closed curves in $M$. Let $R_{1}$ and $R_{2}$ be compact subsets of the plane, bounded by $Q_{1}$ and $Q_{2}$ respectively. One has $R_{1} \subseteq R_{2}$ or $R_{2} \subseteq R_{1}$. W.l.g. let $R_{1} \subseteq R_{2}$, see figure 5 . Denote $M_{1}=R_{1} \cap M$. Notice that $M_{1}$ is a derivation $N$-diagram for $l\left(Q_{1}\right)$. Fix $\phi_{D}: \delta^{\prime}(D) \rightarrow \Gamma(N)$ of Definition 13. The piece $P$ splits in $P_{1}$ and $P_{2}$ in $\delta^{\prime}(D)$, see figure 5. $f\left(\phi_{D}\left(P_{1}\right)\right)=f\left(\phi_{D}\left(P_{2}\right)\right)$ by definition of inessential boundary. Let $v=f\left(\phi_{D}(o(P))\right) \in V . \Gamma(H)$. Take a path $\mathcal{T}$ from $1_{H}$ to $v$ in $\Gamma(H)$. Add a path ("tail") $T$ with $l(T)=l(\mathcal{T})$ to the diagram $M_{1}$. The resulting diagram $M_{1}^{\prime}$ is a derivation $N$-diagram for $l\left(T Q_{1} T^{-1}\right)=u^{\prime} \in H$. There are two possibilities:

i) $u^{\prime} \in \Sigma$

ii) $u^{\prime} \in N$.

In the case i) $r\left(u^{\prime}\right)<r(u)$ and we get a contradiction with the minimality of $r(u)$. Consider case ii). Notice that $l\left(T Q_{1} P Q_{2} P^{-1} T^{-1}\right) \in N$ and, consequently, $l\left(T P Q_{2} P^{-1} T^{-1}\right) \in N$. So, there is a cycle in $\Gamma(N)$ with label $l\left(Q_{2}\right)$. It follows that we may remove $D$ and $R_{1}$, and glue new region $D^{\prime}$ along $Q_{2}$. A new diagram $M^{\prime}$ is a derivation $N$-diagram for $u$ with $r\left(M^{\prime}\right) \leq r(M)-1$, a contradiction.

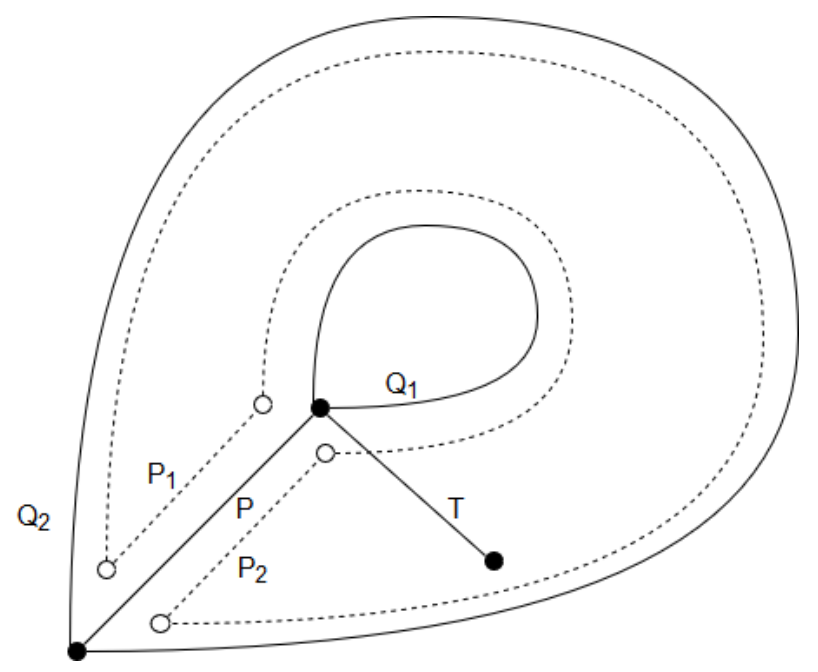

Figure 5: Region with self-boundary

\subsection{Another proof of Theorem 3}

Theorem 3 may be seen as a particular case of Theorem 7.15, [1]. For proof, we need the adapted definition of the distance $\hat{d}$ of [1] only. We do not state the results of [1], the interested reader might choose to look 
at [1].

A product $w_{1} h_{1} \ldots h_{n-1} w_{n}$ is said to be alternating if

I) $h_{i} \in H$

II) $w_{1} h_{1} \ldots w_{i} \notin H$ for $i<n$.

The weight of an alternating product is

$$
\left\|w_{1} h_{1} \ldots h_{n-1} w_{n}\right\|_{H}=\sum_{i=1}^{n}\left(\left|w_{i}\right|+1\right)-1
$$

For $h \in H$ define $\hat{d}(1, h)=\hat{d}_{H}(1, h)$ to be the least $\left\|w_{1} h_{1} \ldots h_{n-1} w_{n}\right\|_{H}$ over all alternating products $w_{1} h_{1} \ldots h_{n-1} w_{n}=h$.

Proposition 9. The length functions $\hat{d}_{H}(1, \cdot)$ and $|\cdot|_{H}$ are Lipshitz equivalent.

Theorem 7.15 of [1] implies an analogue of our Theorem 3, with the length function $|\cdot|_{H}$ replaced by the length function $\hat{d}_{H}(1, \cdot)$, see [1] for details. So, Theorem 3 follows by Proposition 9. The drawback of this approach is the absence of an estimate for $\gamma_{H}$.

\subsubsection{Proof of Proposition 9}

Let a free group $F$ be freely generated by $X$. Let $w=x_{1} x_{2} \ldots x_{n}$ be a reduced word in $X \cup X^{-1}$. We call a prefix $x_{1} x_{2} \ldots x_{i}$ admissible if $P\left(1_{H}, v, x_{1} \ldots x_{i}\right)$ exist for some $v \in V \cdot \Gamma(H)$ (such a path is unique). A prefix $x_{1} \ldots x_{i}$ is a maximal admissible prefix if it is admissible and $x_{1} \ldots x_{i+1}$ is not (or $i=n$ ).

Fix an alternating product $w_{1} h_{1} \ldots w_{n-1} h_{n-1} w_{n}$. Let $a_{i} \circ b_{i}=w_{1} h_{1} \ldots w_{i} h_{i}$, where $a_{i} \circ b_{i}$ is a product without cancellation and $a_{i}$ is a maximal admissible prefix of $a_{i} b_{i}$.

Lemma 11. $\left|a_{i}\right|_{H} \leq \sum_{j=1}^{i}\left(\left|w_{j}\right|+C\right)$, where $C$ is of Lemma .

Proof. Induction on $i$. For $i=0$ there is nothing to prove.

$i \Longrightarrow i+1$. Let $P\left(1_{H}, v_{i}, a_{i}\right)$ be a path in $\Gamma(H)$ and $a_{i+1} \circ b_{i+1}=a_{i} b_{i} w_{i+1} h_{i+1}$. If not all letters of $b_{i}$ cancel with $w_{i+1} h_{i+1}$ then $a_{i+1}=a_{i}$ and proof is concluded by induction. So, let $a_{i+1}=a_{i} \alpha$ with a path $P\left(v_{i}, v_{i+1}, \alpha\right)$. (Notice that cancellations in $a_{i} \alpha$ do not cause problems.) We may represent $\alpha=\alpha_{0} \circ \alpha_{1}$, where $\alpha_{0}$ is a subword of $w_{i+1}$ and $\alpha_{1}$ is a subword of $h_{i+1}$. (Some $\alpha_{i}$ may be empty.) We may decompose $P\left(v_{i}, v_{i+1}, \alpha_{0} \circ \alpha_{1}\right)=P\left(v_{i}, v^{\prime}, \alpha_{0}\right) P\left(v^{\prime}, v_{i+1}, \alpha_{1}\right)$. As $\alpha_{1}$ is a subword of $h_{i+1}$ there is $P\left(u^{\prime}, u, \alpha_{1}\right)$ which is a subpath of $P\left(1_{H}, 1_{H}, h_{i+1}\right)$, i.e..

$$
P\left(1_{H}, 1_{H}, h_{i+1}\right)=P\left(1_{H}, u^{\prime}, h_{i+1}^{\prime}\right) P\left(u^{\prime}, u, \alpha_{1}\right) P\left(u, 1_{H}, h_{i+1}^{\prime \prime}\right),
$$

for corresponding representation $h_{i+1}=h_{i+1}^{\prime} \circ \alpha_{1} \circ h_{i+1}^{\prime \prime}$. We claim that $u^{\prime} \neq v^{\prime}$. Indeed, $a_{i+1}=$ $w_{1} h_{1} \ldots w_{i} h_{i} w_{i+1} h_{i+1}^{\prime} \alpha_{1}$ and there is a path $P\left(1_{H}, v_{i+1}, a_{i+1}\right)$. If $u^{\prime}=v^{\prime}$ then $v_{i+1}=u$ by the uniqueness of the path with given origin and label. So, the reduction of a path $P\left(1_{H}, v_{i+1}, a_{i+1}\right) P^{-1}\left(1_{H}, u=v_{i+1}, h_{i+1}^{\prime} \alpha_{1}\right)$ is a path $P\left(1_{H}, 1_{H}, w_{1} h_{1} \ldots w_{i} h_{i} w_{i+1}\right)$, a contradiction with $\left.\mathbf{I I}\right)$. So, $u^{\prime} \neq v^{\prime}$ and there is a path in $\Gamma(H) \dot{\times} \Gamma(H)$ with label $\alpha_{1}$. It follows that $\left|\alpha_{1}\right|_{H} \leq C$ by Lemma 7. It is also clear that $\left|\alpha_{0}\right| \leq\left|w_{i+1}\right|$.

Taking alternating representation $h=w_{1} h_{1} w_{2} h_{2} \ldots w_{n}$ for $h \in H$ and substituting $i=n$ in Lemma 11 we get the following corollary.

Corollary 5. $|h|_{H} \leq C \hat{d}_{H}(1, h)$ for $h \in H$.

Consider the other inequality. 
Lemma 12. $\hat{d}_{H}(1, h) \leq(1+2 \operatorname{diam}(\Gamma))|h|_{H}$.

Proof. Let $|h|_{H}=k$. Then there is a presentation $h=w_{1} h_{1} w_{2} h_{2} \ldots h_{n-1} w_{n}$ with $1 \neq h_{i} \in H^{a_{i}} \cap H^{a_{i}^{\prime}}$ and

$k=\sum_{i=1}^{n}\left(\left|w_{i}\right|+1\right)-1$. W.l.g. $\left|a_{i}^{\prime}\right|,\left|a_{i}\right| \leq \operatorname{diam}(\Gamma(H))$. Define an alternating product $h=w_{1}^{\prime} h_{1}^{\prime} \ldots w_{n}^{\prime}$ where $h_{i}^{\prime}=b_{i} h_{i} b_{i}^{-1}$ and $w_{i}^{\prime}=b_{i-1} w_{i} b_{i}^{-1}, b_{0}=b_{n}=1$. Here $b_{i}=a_{i}$ or $a_{i}^{\prime}$ is chosen in such a way that $w_{1}^{\prime} h_{1}^{\prime} \ldots w_{i}^{\prime} \notin H$ for $i<n$. Clearly, $\left|w_{i}^{\prime}\right| \leq\left|w_{i}\right|+2 \operatorname{diam}(\Gamma)$.

Notice that Corollary 5 and Lemma 12 imply Proposition 9.

\section{References}

[1] F. Dahmani, V. Guirardel, and D. Osin, "Hyperbolically embedded subgroups and rotating families in groups acting on hyperbolic spaces," ArXiv e-prints, Nov. 2011.

[2] A. Y. Ol'shanskii, "The sq-universality of hyperbolic groups," Sbornik: Mathematics, vol. 186, no. 8, pp. 1199-1211, 1995.

[3] B. H. Neumann, M. P. Murthy, B. H. Neumann, and B. H. Neumann, Lectures on topics in the theory of infinite groups, vol. 21. Tata Institute of Fundamental Research, 1968.

[4] J. R. Stallings, "Surfaces in three-manifolds and non-singular equations in groups," Mathematische Zeitschrift, vol. 184, no. 1, pp. 1-17, 1983.

[5] D. V. Osin, "Peripheral fillings of relatively hyperbolic groups," Invent. Math., vol. 167, no. 2, pp. 295326, 2007.

[6] G. Higman, B. H. Neumann, and H. Neumann, "Embedding theorems for groups," J. London Math. Soc., vol. 24, pp. 247-254, 1949.

[7] B. B. Newman, "Some results on one-relator groups," Bull. Amer. Math. Soc., vol. 74, pp. 568-571, 1968.

[8] R. C. Lyndon and P. E. Schupp, Combinatorial group theory. Classics in Mathematics, SpringerVerlag, Berlin, 2001. Reprint of the 1977 edition.

[9] M.-P. Schützenberger, "Sur l'équation $a^{2+n}=b^{2+m} c^{2+p}$ dans un groupe libre," C. R. Acad. Sci. Paris, vol. 248, pp. 2435-2436, 1959.

[10] R. C. Lyndon and M. P. Schützenberger, "The equation $a^{M}=b^{N} c^{P}$ in a free group," Michigan Math. J., vol. 9, pp. 289-298, 1962.

[11] I. Kapovich and A. Myasnikov, "Stallings foldings and subgroups of free groups," J. Algebra, vol. 248, no. 2, pp. 608-668, 2002.

[12] F. Levin, "Solutions of equations over groups," Bull. Amer. Math. Soc., vol. 68, pp. 603-604, 1962.

[13] O. Bogopolski, Introduction to group theory. EMS Textbooks in Mathematics, European Mathematical Society (EMS), Zürich, 2008. Translated, revised and expanded from the 2002 Russian original. 